\title{
Targeting Neural Hyperactivity as a Treatment to Stem Progression of Late-Onset Alzheimer's Disease
}

\author{
Rebecca P. Haberman ${ }^{1}$ - Audrey Branch ${ }^{1}$ - Michela Gallagher ${ }^{1}$
}

Published online: 30 May 2017

(C) The Author(s) 2017. This article is an open access publication

\begin{abstract}
Sporadic late-onset Alzheimer's disease (LOAD), the most common form of dementia in the elderly, causes progressive and severe loss of cognitive abilities. With greater numbers of people living to advanced ages, LOAD will increasingly burden both the healthcare system and society. There are currently no available disease-modifying therapies, and the failure of several recent pathology-based strategies has highlighted the urgent need for effective therapeutic targets. With aging as the greatest risk factor for LOAD, targeting mechanisms by which aging contributes to disease could prove an effective strategy to delay progression to clinical dementia by intervention in elderly individuals in an early prodromal stage of disease. Excess neural activity in the hippocampus, a recently described phenomenon associated with age-dependent memory loss, was first identified in animal models of aging and subsequently translated to clinical conditions of aging and early-stage LOAD. Critically, elevated activity was similarly localized to specific circuits within the hippocampal formation in aged animals and humans. Here we review evidence for hippocampal hyperactivity as a significant contributor to agedependent cognitive decline and the progressive accumulation of pathology in LOAD. We also describe studies demonstrating the efficacy of reducing hyperactivity with an initial test therapy, levetiracetam (Keppra), an atypical antiepileptic. By
\end{abstract}

Electronic supplementary material The online version of this article (doi:10.1007/s13311-017-0541-z) contains supplementary material, which is available to authorized users.

Rebecca P. Haberman

rahabs@jhu.edu

1 Department of Psychological and Brain Sciences, The Johns Hopkins University, 3400 North Charles Street, 116 Dunning Hall, Baltimore, MD 21218, USA targeting excess neural activity, levetiracetam may improve cognition and attenuate the accumulation of pathology contributing to progression to the dementia phase of LOAD.

Keywords Cognitive aging - Levetiracetam (Keppra) · Late-onset Alzheimer's disease · Hippocampal CA3 . Amnestic mild cognitive impairment (aMCI)

\section{Introduction}

Sporadic late-onset Alzheimer's disease (LOAD), the most common form of dementia, is heralded by an insidious and progressive erosion of episodic memory in the later decades of life. Longitudinal observational studies have confirmed the existence of a symptomatic prodromal phase of LOAD, referred to as amnestic mild cognitive impairment (aMCI), in which memory deficits are greater than would be expected for a person's age but do not significantly impair activities of daily life. This condition substantially increases risk for progression to the protracted phase of Alzheimer's disease (AD) dementia during which deterioration continues until all cognitive domains are severely compromised $[1,2]$.

Investigations using disease biomarkers have now identified a prolonged asymptomatic stage of LOAD in which the accumulation of extracellular amyloid plaques can be detected by positron emission tomography (PET) neuroimaging in elderly individuals who are clinically normal [3]. Thus, the hallmark accumulation of pathological plaques containing aggregated forms of amyloid $\beta$ (A $\beta$ ) peptide occurs prior to the advancing spread of neurofibrillary tangles composed of hyperphosphorylated tau and widespread neurodegeneration. Preclinical amyloid deposition, alongside the discovery of genetic links between $A \beta$ mutations and risk for early-onset familial $\mathrm{AD}$ (FAD) $[4,5]$, has focused biomedical research 
and therapeutic strategies on reducing or reversing $A \beta$ deposition. However, several high-profile failures of LOAD clinical trials targeting $A \beta$ in patients with early- to mid-stage dementia have repositioned the timing of $A \beta$ intervention to earlier disease stages and broadened the scope of therapeutic strategies to include alternative stage-specific targets [6-8]. Despite the fact that the clinical diagnosis of LOAD typically peaks in the seventh and eighth decades of life, it is often overlooked in therapeutic development programs that aging itself constitutes the single greatest risk factor for LOAD [9]. The aging context in which the long preclinical phase of pathophysiological development is situated has promoted greater attention to mechanisms by which brain aging may confer risk for progression of underlying pathology and symptomatic disease.

An emerging view of LOAD is that age-dependent alterations in neuronal activity act to promote the progression of memory loss and the accumulation of AD pathology. While neuron loss and hypoactivity are key features of later disease stages [10,11], human neuroimaging studies, including those in elderly patients with aMCI, have identified a paradoxical hyperactivity signature in the hippocampus [12-16]. Initially thought to be a compensatory mechanism to support memory function, studies in elderly individuals and patients with aMCI have demonstrated that hippocampal hyperactivity is actually correlated with reduced cognitive performance within those populations [14, 17-20]. Significantly, pharmacological reduction of this overactivity in aMCI has demonstrated that it contributes to memory impairment rather than serving a beneficial role [20,21]. Animal models of normal aging have also indicated that hippocampal hyperactivity is associated with memory impairment and that its natural absence in aged cohorts coincides with preserved cognition and pharmacological reversal in aged animals with cognitive impairment restores memory function [22-26].

Transgenic models designed to recapitulate the hallmark pathological features of AD have increasingly implicated excess neural activity as a causal and/or permissive factor in initiation and progression of $A \beta$ and tau pathology. Young mice overexpressing human amyloid based on the genetics of early-onset FAD have shown that neuronal circuits become hyperactive in early stages of amyloid accumulation, contributing to neuronal injury [27-29]. Reduction of neural activity in such models produces beneficial effects on synaptic dysfunction and reduces amyloid deposition in some AD models [30]. Moreover, recent evidence from AD models of human tau mutations has demonstrated that chronically increased neural activity stimulates the release of tau and enhances the spread of tau pathology in the hippocampus and associated circuits [31]. Together with the emergence of circuit-specific hyperactivity in aging, these studies suggest that alterations in neural excitability may constitute an underlying basis that contributes to the risk of aging in late-onset $\mathrm{AD}$ progression.
In this review, we present evidence from both human clinical studies and animal models linking age-related hyperactivity with the worsening cognitive impairment found in early disease stages, as well as the accumulation of AD neuropathology. Further, we discuss therapeutic strategies for targeting age-related hyperactivity in early $\mathrm{AD}$, with a focus on low-dose levetiracetam (LEV), an atypical antiepileptic that has shown efficacy in selectively reducing aberrant, but not basal, neural activity, resulting in improved cognitive outcomes in preclinical and clinical studies. Together these findings provide support for the hypothesis that aberrant neural activity in the aging brain may represent an underlying basis of risk for late-onset sporadic $\mathrm{AD}$, and is a potential therapeutic target for delaying or preventing disease progression in the earliest stages of LOAD prior to clinical dementia.

\section{Hyperactivity in the Hippocampal Memory System is Localized to Key Computational Circuits in Normal Aging}

The aging-specific, biological phenotype that confers risk for LOAD is difficult to separate from pathological processes in elderly humans owing to the lengthy preclinical phase of the disease superimposed upon an aging brain [3] and substantial variability in cognitive trajectories/outcomes across the aging human population. Studies of primates and rodents that do not evince overt $\mathrm{AD}$ pathology are informative for isolating changes due to brain aging apart from the pathological processes in $\mathrm{AD}$. Consistent with individual differences evident in the normal aged human population, memory assessment also reveals individual differences in aged animal populations [32-34]. In aged rodents and monkeys with memory impairment, hyperactive neurons with elevated firing rates have been specifically localized to the CA3 subregion of the hippocampus in a manner consistent with the degree of memory impairment $[23,35]$. In that context, studies using functional magnetic resonance imaging (fMRI) with high neuroanatomical resolution within the medial temporal lobe (MTL) have similarly localized excess age-related activation during memory performance to the dentate gyrus (DG) and CA3 (DG/CA3) subregions of the hippocampal formation in humans [18]. That excess activation detected by fMRI, as discussed further below, is also correlated with the degree of memory impairment in the elderly.

The contribution of the MTL system to episodic memory critically depends on the computational properties of the DG and CA3 subregions of the hippocampal formation of the MTL (Fig. 1). Layer 2 entorhinal cortex (EC) projections provide the primary input to the hippocampal network to encode the content of current experiences [36]. The normal function of the DG and CA3 subregions establishes distinctive representations in memory that minimize interference from similar 


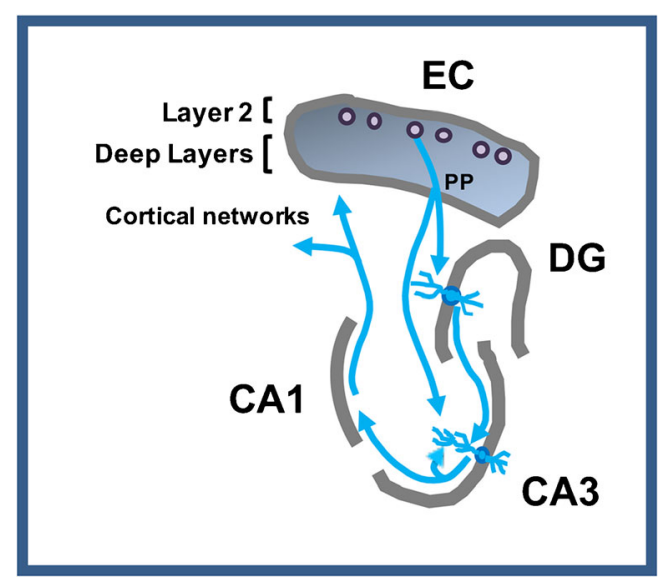

Fig. 1 Schematic of connectional pathways. The perforant path (PP) from entorhinal cortex (EC) layer 2 cells provides innervation of the dentate gyrus (DG) and CA3. CA3 pyramidal neurons also receive synaptic input from DG mossy fibers and CA3 recurrent collaterals. The output of the DG/CA3 computational processing occurs through the CA1 subfield and the subiculum (not shown) projecting to deep layers of the EC and additional cortical structures

experiences in the past. This ability to encode and retrieve information tied to specific events and experiences is critical for proficient episodic memory. The minimization of interference is normally implemented in the DG by "pattern separation", referring to the highly distinctive encoding of input in a sparse network of granule cells, even when the input pattern has overlapping elements with prior information [37]. Encoding in the CA3 region, which receives its input from the DG, is also influenced by its strong autoassociative network, which retrieves similar representations from prior encoding by a process referred to as "pattern completion" [38]. These competing yet complementary processes are thought to minimize interference while increasing storage capacity for episodic memories [39, 40]. Computational models and empirical studies on the normal function of these circuits in laboratory animals have translated to normal young adults using neuroimaging methods [41, 42]. As reviewed in further detail elsewhere [43, 44], evidence for an effect of aging on the functional properties within this network occurs in agerelated memory impairment in nonhuman species, as well as humans.

In aged rodents with memory impairment the balance of pattern separation and pattern completion within this network is shifted to diminish pattern separation in favor of pattern completion. Specifically, the encoding properties of neurons in this network of memory-impaired aged rats fail to rapidly develop distinctive representations that reflect pattern separation but instead exhibit interference in the retrieval of prior representations $[26,35]$. Elevated neural activity directly recorded from CA3 neurons in aged memory-impaired animals in these studies likely contributes to this dysfunction in the balance of computational properties of the network [43]. Similarly, localization of excess fMRI activation in DG/CA3 region is observed in elderly humans, and likely also contributes to heightened interference in episodic memory $[42,44]$.

In tasks specifically designed to capture the key computational functions just described in humans, aged individuals are prone to mnemonic interference, exhibiting a shift in memory performance attributable to $\mathrm{DG} / \mathrm{CA} 3$ dysfunction similar to that described in aged memory-impaired animals. To assess the capacity for pattern separation in humans, a recognition memory task using 3 judgments has now become widely used in neuropsychological research on aging and prodromal $\mathrm{AD}$ $[45,46]$. In one common version of this task subjects are asked to judge whether pictures of everyday objects presented in a series are "new" (viewed for the first time), "old" (a repeat of a previous item in the series), or "similar" (resembling but not identical to a previous item in the series). A correct response of "similar" requires pattern separation to reduce mnemonic interference. Compared with young adults, older adults have less proficiency in the correct identification of items that are "similar" to a previously viewed item, instead calling such items "old", an error indicative of a shift towards pattern completion [46-49]. High-resolution fMRI conducted during performance of the 3-judgment task showed greater hippocampal activation in the DG/CA3 region in elderly subjects compared with young adults alongside significantly worse performance, with fewer correct responses of "similar" and greater incorrect responses of "old" on those items [18]. Moreover, worse discrimination performance was significantly correlated with relatively greater DG/CA3 hippocampal activation.

A number of the studies assessing mnemonic interference in cognitively normal young and aged adults have highlighted individual differences in the elderly population. Notably, the subgroups of aged individuals with less proficient performance in 3judgment recognition have corresponding differences in performance on standardized memory testing for delayed recall [46]. Individual differences are also common in aging across species. As a model of memory loss in normal aging, outbred rats show variability in cognitive outcomes such that a subgroup shows memory decline relative to young performance, while other aged cohorts exhibit preserved performance on par with young [33, 50]. These subgroups of "impaired" and "unimpaired" aged rats also differ in the encoding characteristics of neurons in the DG/ CA3 network as already described. In recordings from ensembles of hippocampal neurons, similar to young adult rats, aged rats that are behaviorally characterized as unimpaired in memory performance exhibit rapid encoding of distinctive representations. In contrast memory-impaired aged rats, unlike young adult and unimpaired aged rats, fail to rapidly encode distinctive representations for new experiences, instead activating prior representations [26]. Individual differences in the heightened CA3 excitability associated with memory impairment in this model is evident by direct neural recordings [35]. This finding was replicated in a recent study using a pharmacological stimulus to induce neuronal activation and detection of activity by 
expression of the immediate early gene, cFos (Fig. 2A) [22]. cFos was increased in aged memory-impaired rats relative to both young and aged unimpaired rats, exhibiting a tight correlation with memory status such that greater cFos expression in CA3 coincided with poorer memory among the animals in the aged cohort.

In summary, memory impairment in both aged rats and aged humans is associated with neuronal hyperactivity similarly localized to the DG/CA3 subfields, with evidence from cognitive assessment indicating corresponding shifts in the balance of computational functions in hippocampal processing.

\section{An Augmented Detrimental Effect on the Computational Functions of the Hippocampal System in aMCI}

Numerous studies have now demonstrated that hippocampal hyperactivity is augmented in patients with aMCI when compared with age-matched control subjects $[10,15,20,21$, 51-55], and is a highly consistent and characteristic signature in aMCI. This hyperactivity is also a measure that predicts subsequent cognitive decline/conversion to a dementia diagnosis [12-14] and is significantly correlated with the extent of neuronal injury affecting AD-specific regions of the aMCI brain [17]. Moreover, hippocampal hyperactivity is most pronounced in $\mathrm{MCI}$ associated with $\mathrm{AD}$ pathology, as determined by PET amyloid imaging [56]. Furthermore, that hyperactivity persists in the MCI phase of the disease over a 3-year followup, during which time greater clinical/cognitive worsening is evident in amyloid positive patients with MCI, relative to patients with amyloid negative PET scans.

Using high-resolution fMRI to localize differences in activation within the MTL in prodromal $A D$, studies have consistently reported elevated activation in the DG/CA3 subregions in aMCI relative to age-matched cognitively normal controls (Fig. 3) [15, 20, 21, 54]. Together with that regionally defined augmentation of fMRI activation, the profile in age-related memory impairment in elderly humans is also significantly magnified in patients with aMCI. Relative to age-matched controls, the performance of patients with aMCI on 3judgment tasks shows a further worsening in the ability to make correct responses to "similar" items while committing more errors in identifying such items as "old" $[20,21,46]$. The further heightening of fMRI activation in the DG/CA3 subregions of the hippocampus is tightly correlated with this worsening performance $[15,20]$.

The clinical condition of aMCI is characterized by a symptomatic worsening of memory performance beyond that considered normal in the aging population. The studies just described in aMCI tie this condition to alterations in the specific circuits and cognitive processes that are also vulnerable in aging. These commonalities may indicate that age-related memory impairment and the transition to the early symptomatic phase of $\mathrm{AD}$ exist on a continuum. In that context, a recent report directly links the existence of hyperactivity to the accumulation of AD pathology in humans. In the study by Leal et al. [57] cognitively normal elderly subjects received neuroimaging with fMRI at baseline and were followed for 3 years with longitudinal PET imaging for amyloid. Hippocampal
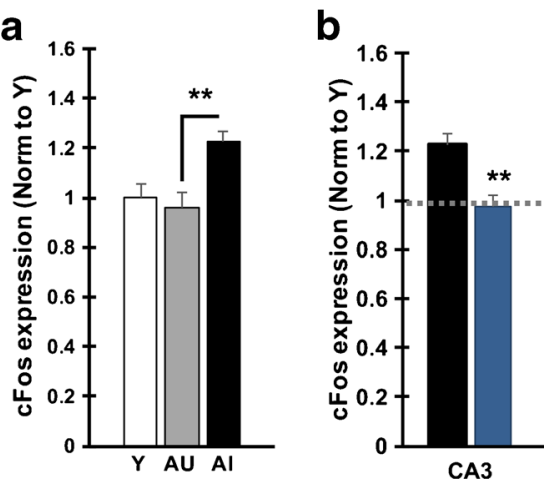

Fig. 2 Pharmacologically induced neural activity is elevated in aged rats with memory impairment and reduced by levetiracetam (LEV) treatment. Neural activity was pharmacologically induced (pilocarpine, $25 \mathrm{mg} / \mathrm{kg}$ i.p.) in young (Y), aged rats with intact memory (aged unimpaired, $\mathrm{AU}$ ) and aged impaired rats (AI). Induced neural activity was detected by quantification of cFos mRNA by in situ hybridization of brain sections collected $1 \mathrm{~h}$ after induction. (A) CA3 subfield of the hippocampus shows higher expression of $\mathrm{cFos}$ in $\mathrm{AI}$ rats compared with $\mathrm{Y}$ and $\mathrm{AU}$ rats. cFos expression also correlates with a measure of memory impairment among all aged rats (Pearson $r=0.832$ ). AI rats also showed increased cFos in retrosplenial (RSC) and parietal cortex (PC) relative to both $\mathrm{AU}$ and $\mathrm{Y}$ rats (data not shown). (B) AI rats in the LEV condition (AI-LEV) were treated for 1 month prior to pilocarpine administration

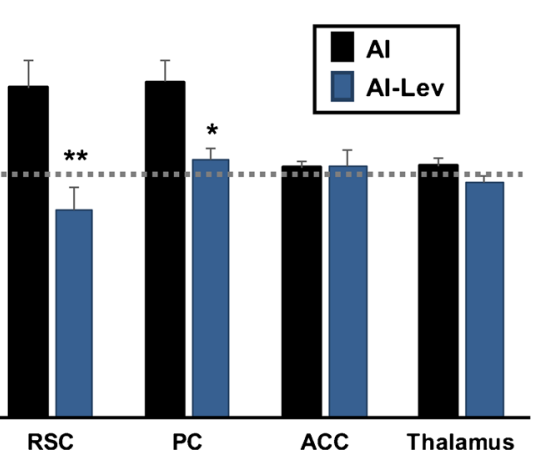

with LEV via osmotic pumps $(10 \mathrm{mg} / \mathrm{kg} / \mathrm{day})$. Treatment with LEV reduced $\mathrm{cFos}$ expression in $\mathrm{AI}$ rats in the $\mathrm{CA} 3$ region of the hippocampus and select interconnected regions, including RSC and PC. ACC and thalamus show no impairment-dependent elevation of cFos and no reduction with LEV treatment. Significant difference across groups was determined by 1 -way analysis of variance. Post-hoc significance was determined by $t$ test as indicted on the graphs: $* p<0.05$; * $p<0.01$. Values represent group means $\pm \mathrm{SEM}$. Gray dotted line represents $\mathrm{cFos}$ expression in young rats. Figure adapted from Neurobiology of Aging, Haberman, RP, Koh, MT, and Gallagher, M, Heightened Cortical Excitability in Aged Rodents with Memory Impairment, in press, Copyright 2017) and reprinted with permission from Elsevier [22]. $\mathrm{ACC}=$ anterior cingulate cortex 


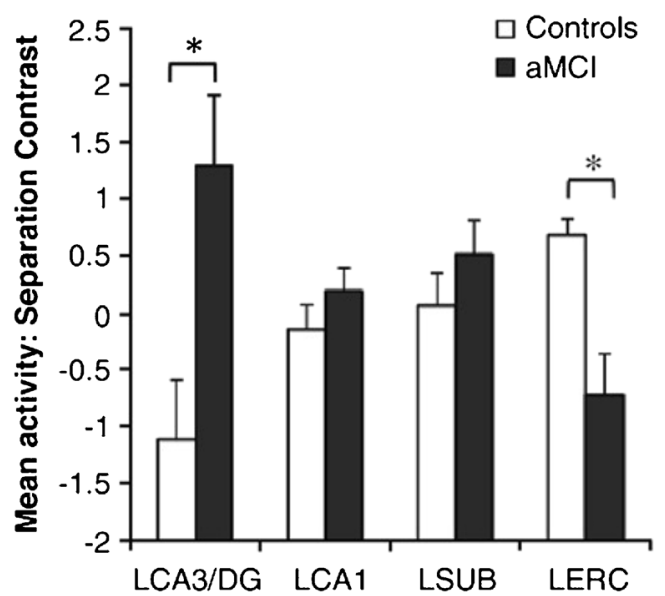

Fig. 3 High-resolution functional magnetic resonance imaging signals in amnestic mild cognitive impairment (aMCI) vs age-matched controls. Patients with aMCI had significantly higher task-related activation in the left CA3 (LCA3)/dentage gyrus (DG) together with lower activity in the left entorhinal cortex (LERC) during encoding for similar items on 3-judgement task. * aMCI significantly different from controls. Figure reprinted from Neuroimage, 51, Yassa, MA, Stark, SM, Bakker, A, Albert, MS, Gallagher, M and Stark, CEL, High-resolution structural and functional MRI of hippocampal CA3 and dentate gyrus in patients with amnestic Mild Cognitive Impairment, 1242-1252, Copyright (2010) with permission from Elsevier [15]. LCA1 $=$ left CA1; LSUB $=$ left subiculum

hyperactivity in the study group at baseline predicted the accumulation of $\mathrm{AD}$ pathology.

\section{Reduction of Hippocampal Hyperactivity with LEV has Therapeutic Efficacy in Aging and aMCI}

The functional significance of the association between hippocampal hyperactivity and MTL-dependent memory deficits in aging and aMCI was initially subject to differing interpretations. According to one view, elevated hippocampal activity detected by fMRI during mnemonic tasks was interpreted as a compensatory mechanism to support memory function by recruiting a greater response in a failing network [10]. Alternatively, increased activation might reflect a dysfunctional condition contributing to memory impairment by shifting hippocampal computational processing as described above. Experimental evidence subsequently obtained by targeting hippocampal hyperactivity with treatments that reduce activity demonstrated support for the latter view that overactivity, detected by fMRI in humans and multiple measures of elevated neural activity in animals, contributes to memory impairment.

\section{Targeting Hyperactivity Improves Memory Performance in Aged Rats}

Research in aged cognitively-impaired rats first tested the hypothesis that CA3 hyperactivity served to impair cognition.
Koh et al. [24] targeted CA3 hyperactivity both locally, using virally transduced inhibitory neuropeptides, and systemically, with peripheral administration of low-dose atypical antiepileptics, valproate, and LEV. Both local and systemic treatments resulted in cognitive improvement in aged impaired rats on 2 independent spatial memory paradigms that critically depend on intact MTL function. A LEV dose-response curve demonstrated efficacy in a low-dose range ( 5 and $10 \mathrm{mg} / \mathrm{kg}$ ), significantly lower doses than required for antiepileptic efficacy in seizure models.

The cognitively effective dose of LEV that restored memory in aged impaired rats has been used in other protocols that demonstrate heightened excitability of hippocampal neurons. Pharmacological induction of neural activity detected by elevated cFos expression in the hippocampal CA3 subfield in aged rats with memory impairment is reduced by LEV treatment (Fig. 2B). With LEV treatment, cFos expression in the aged impaired rats remained similar to levels expressed in young rats and aged rats with preserved cognition [22]. In addition, multiunit recording of hippocampal CA3 neurons also showed reduction of neuronal firing rates in response to targeting overactivity with systemic drug treatment [58]. These investigations demonstrate that targeting aberrant excess hippocampal activity is an effective strategy for improving memory in aged rats with impairment.

A second effective preclinical approach to targeting overactivity in aged impaired rats has focused on the use of positive allosteric modulators of $\gamma$-aminobutyric acid (GABA)$\mathrm{A} \alpha 5$ receptors. These receptors normally mediate tonic inhibition and have exceptionally high expression localized to the hippocampus relative to other brain regions [59]. Negative allosteric modulators of GABA-A $\alpha 5$, which increase hippocampal excitability, were previously reported to modestly increase performance of young rats in spatial memory paradigms [60-63]. In contrast, the use of a negative allosteric modulator showed no such beneficial effect in memoryimpaired rats with a condition of hippocampal overactivity. Instead, positive allosteric modulators of GABA-A $\alpha 5$ receptors, which reduce neural excitability, have been shown to improve memory performance in aged memory-impaired rats [25]. The high expression of GABA-A $\alpha 5$ receptors on CA3 pyramidal neurons makes them ideally positioned to control the overactivity of those neurons. In addition, recent experimental evidence has demonstrated a contribution of GABA$A \propto 5$ receptors in the DG to pattern separation [64]. Removal of DG GABA-A $\alpha 5$ receptors reduced tonic, but not phasic, inhibition and elevated the response in DG to a stimulating input. Elevated DG activity was coupled to impaired performance on behavioral tests of pattern separation, consistent with a contribution of hyperactivity to impaired DG/CA3 computational processing.

Together, these findings support the conclusion that elevated hippocampal activity serves to impair hippocampal 
function in aged rats. While the use of selective GABA-A $\alpha 5$ positive allosteric modulators are a promising therapeutic approach currently in preclinical development, given the long history of LEV safety at much higher doses to treat patients with epilepsy [65-67], this treatment was used in clinical studies of patients with aMCI to determine whether targeting hippocampal hyperactivity would show translational evidence for benefit.

\section{LEV Reduction of Hippocampal Hyperactivity in aMCI Improves Memory Performance}

Successful treatment in aged impaired rats led to clinical testing of LEV in elderly patients with aMCI [20]. Mechanistic studies of LEV action suggest it is a promising proof-ofconcept drug to examine the clinical outcomes of reducing excess activity. By binding to the synaptic vesicle protein, SV2a, LEV selectively dampens neurotransmitter release under conditions of elevated firing, but not baseline neurotransmission [68-70].

To assess the effect of LEV on elevated activity and behavior in patients with aMCI, a low dose (125 mg q12h), equivalent to an efficacious dose in rodents, was initially tested. A double blind, within-subject crossover design allowed the comparison of an individual's memory performance with and without LEV treatment [20]. Memory was tested in the 3 -judgement memory task as previously described, and concurrent $\mathrm{fMRI}$ scans confirmed task-specific excess activation of DG/CA3 subdivision of the hippocampus. LEV treatment significantly reduced DG/CA3 activation in patients with aMCI and significantly improved their performance on the 3-judgment memory task, increasing correct responses of similar (indicative of improved pattern separation) while committing fewer errors of "old" responses to those items. The improvement of patient performance under treatment that reduced hippocampal hyperactivity provided the first evidence that fMRI overactivity in the hippocampus is not a beneficial signature for compensatory function but rather represents a condition contributing to impairment.

Using a range of dosing regimens in independent cohorts of patients with aMCI, LEV's ability to reduce hyperactivity in the hippocampus with improvement in memory task performance was confirmed [21]. Notably, LEV only improved task performance at doses that reduced the elevated fMRI signal in DG/CA3 [21]. The three cohorts of patients were treated with different doses of LEV, which were selected to achieve brain exposures similar to those shown to have efficacy (low doses) or lack of efficacy (high dose, $250 \mathrm{mg} \mathrm{q} 12 \mathrm{~h}$ ) in preclinical models. In the within-subject crossover design, each aMCI cohort on placebo exhibited significantly increased DG/CA3 hippocampal activation when compared with healthy agematched controls and was similarly impaired in task performance relative to normal aging. Low doses in the range of efficacy observed in the preclinical research significantly improved memory task performance and normalized fMRI signals so that no difference remained in comparison with agematched controls. In addition, similar to the results observed in memory-impaired aged rats, therapeutic efficacy was lost at a higher drug exposure that remains below the range used therapeutically in epilepsy. These data provide strong evidence that LEV reduction of excess neural activity in the hippocampus provides therapeutic benefit and demonstrates that hippocampal hyperactivity contributes to impaired memory rather than performing a compensatory role.

It has long been recognized that the MTL system is vulnerable both in aging when memory complaints become quite common and also in the early pathological progression of $\mathrm{AD}$. The fact that both aging and AD involve common specific circuits within this system has become better defined in relatively recent research. It is not yet clear, however, whether these parallels reflect an underlying high vulnerability of specific circuits to a variety of conditions acting independently, aging on the one hand and the pathophysiological insults of $\mathrm{AD}$ on the other, or whether the condition of aging itself contributes to the vulnerability and progression of disease [71].

\section{Neural Hyperactivity and AD Pathophysiology}

As discussed in previous sections, heightened neural activity is found in both normal aging and in the preclinical and aMCI phases of AD. Transgenic rodent models of AD have been created and studied by overexpressing rare human mutations in the amyloid precursor protein (APP) and associated secretase components (Psen 1 and Psen2) identified in earlyonset FAD and tau mutations causal for frontotemporal dementia $[72,73]$. While rodents do not naturally develop AD pathology, the exposure of the murine brain to high levels of $\mathrm{A} \beta$ results in $\mathrm{AD}$-like changes, including amyloid plaques, neuritic dystrophy, gliosis, synaptic deficits, and a range of cognitive and noncognitive behavioral alterations [74-80]. Similarly, mouse lines carrying frontotemporal dementia tau mutations display neuronal and synaptic dysfunction, inflammatory responses, and axonal degeneration [81-85]. While no AD model perfectly recapitulates the spectrum of human AD progression, these models provide an opportunity to identify the contribution of individual pathological disease factors in vivo, as well as settings for proof-of-concept tests of therapeutic strategies. Increasing evidence from such models indicates an interrelationship between AD pathology and neural activity, strongly supporting hyperactivity as central to the pathological mechanisms of $\mathrm{A} \beta$ and tau.

Most mouse models for AD are based on the amyloid hypothesis of AD progression (reviewed in [86]). The major protein component of amyloid plaques found in $\mathrm{AD}$ is a small 39 to 42-amino acid polypeptide called $\mathrm{A} \beta$, which is derived from 
the proteolytic cleavage of the transmembrane localized APP [5]. $\mathrm{A} \beta$ cleavage results in its release into the extracellular space where it interacts with a variety of receptors, and when present at high levels, aggregates into toxic amyloid plaques. Numerous mutations have been identified in APP and enzymes which regulate its cleavage, the molecular details and effects of which have been extensively reviewed elsewhere $[4,5]$. Pathogenic mutations associated with FAD cause either an increase in the total levels $A \beta$, or modify its cleavage such that it is biased towards its longer form $A \beta_{42}$, which is more strongly associated with the formation of plaques than the shorter $\mathrm{A} \beta_{40}[4,87,88]$. Transgenic mouse models based on these mutations have high levels of $A \beta$ and develop amyloid plaque pathology similar to that seen in human $\mathrm{AD}$, and display behavioral deficits in learning and memory tests $[73,89]$.

Evidence for hyperactivity associated with $A \beta$ was first documented in transgenic mouse models of $\mathrm{AD}$ that overexpress human mutant APP via strong exogenous promoters. EEG recording in freely behaving young adult hAPP-J20 mice showed widespread elevated, but subconvulsive activity in the hippocampus and cortex, which was accompanied by impaired synaptic plasticity mechanisms and other evidence of neuronal injury [77, 90]. Epileptiform activity has since been observed in several transgenic models [91-93], as have spontaneous epileptic seizures [94, 95], which are attributable to increased network hyperexcitability $[91,96]$. The relationship between this aberrant activity and $A \beta$ began to be elucidated in separate studies of plaque-bearing double transgenic mice (APP23 $\times$ PS45) mice carrying APP and PSEN1 mutations where increased numbers of hyperactive neurons were identified in proximity to amyloid plaques across cortical networks including the frontal cortex [97], primary visual cortex [98], and the hippocampal $C A 1$ subfield [27], suggesting that $A \beta$ aggregates may induce higher neural activity. This phenomenon was further confirmed in APPswe/PS1d9 animals, carrying slightly different mutations, using activity dependent reporters [99] and whole-cell patch clamp recordings to demonstrate elevated neural activity alongside dendritic abnormalities [100]. Intriguingly, a subsequent study of APP23 $\times$ PS45 mice indicated that the number of hyperactive neurons present in the CA1 of the hippocampus was substantially elevated at an age prior to plaque deposition relative to controls [27]. This heightened activity was blocked in transgenic animals following reduction of soluble $A \beta$ levels with a $\gamma$-secretase inhibitor, and could be induced in wild-type animals by local application of soluble $A \beta$ oligomers, providing strong evidence for a role of soluble $A \beta$ species as the primary toxic driving force for this hyperactivity, rather than already deposited plaques [29].

The hyperactivity signature associated with familial genetics in these animal models and early-onset FAD may differ in some respects from that observed in aging and prodromal LOAD. A condition of pathological neural hypersynchrony is associated with elevated network epileptiform activity in several of the FAD mouse models. Aberrant epileptiform activity, possibly driven by this hypersynchrony, also appears to be more common clinically in early-onset FAD [101, 102] than in LOAD [103]. A basis for heightened neural activity associated with aging that is nonepileptiform would be consistent with a lower risk for seizures in LOAD relative to early-onset FAD. Nonetheless, irrespective of such differences, evidence across the spectrum of AD suggests a bidirectional effect of $A \beta$ and neural activity.

A recent study builds on the earlier findings that regulation of neural activity modulates $A \beta$ production $[29,104]$. Using viralmediated expression of exogenous receptors (DREADDS) [105], Yuan et al. [30] introduced chronic intermittent increases or decreases in neural activity in APP-overexpressing mice. Strikingly, increases in $A \beta$ levels and deposition were observed following elevation of neural activity, while activity reduction resulted in decreased $A \beta$ accumulation, as well as reduced axonal dystrophy and synaptic loss in areas nearby amyloid plaques. While these studies do not exclude the possibility that other factors may contribute to the pathological effects of $A \beta$, they provide support for the existence of a feed-forward induction loop between $A \beta$ and neural activity. This circular relationship suggests that either elevated neural activity or elevated $A \beta$ may initiate a cascade towards increasing hyperactivity and peptide overproduction leading to amyloid deposition, and, importantly, that restoring balanced activity can ameliorate $\mathrm{A} \beta$ toxicity.

Similar to aged rats and patients with aMCI, therapeutic targeting of neural activity in AD rodent models has been demonstrated to improve cognition and signatures of neuronal injury. In the hAPP-J20 model, administration of LEV improved behavioral performance across several tasks [106], and reduced signatures of neuronal injury [90]. Additional behavioral and neurophysiological efficacy has been shown in other AD models in which amyloid is associated with neuronal hyperactivity [107-110]. Consistent with the LEV mechanisms of selectively targeting aberrant activity, the effects in mutant APP mice produced by LEV (e.g., brain markers and behavior) were not observed in control nontransgenic (nonamyloid) mice given the same drug treatment. Furthermore, a spectrum of other antiepileptic drugs, with mechanisms of action that differ from the atypical antiepileptic LEV, have been ineffective in APP models, including ethosuximide, gabapentin, phenytoin, pregabalin, and vigabatrin $[106,110]$. In that context, it is also notable that doses of LEV that are effective in vivo in preclinical $\mathrm{AD}$ models have been consistently lower than those required for antiseizure efficacy in epilepsy models.

Many effects in mutant APP mice are prevented by removal of endogenous tau $[86,111]$. The regulation of excitability is a leading explanation for the neuroprotection conferred by tau reduction, including a role for endogenous tau in early synaptic pathology and in the toxicity observed in mouse models based on the P301Ltau human tau mutation [112, 
113]. In addition to limiting hyperactivity in mutant APP mice, the reduction of endogenous tau prevented cognitive impairment, synaptic/molecular dysfunction, and neuronal injury in a manner similar to low-dose LEV treatment $[90,93$, $106,111]$. More recently, tau was shown to be required for an increase in hippocampal neuron dendritic excitability initiated by $A \beta$; here tau removal was also mimicked by LEV which prevented both potassium channel depletion and dendritic hyperexcitability [107]. Because a loss of synaptic integrity includes dendritic structural degeneration caused by hyperexcitability [100], such early neurodegeneration could be limited by LEV.

The biological evidence from AD mouse models supports a relationship between neural activity and $\mathrm{AD}$ pathology that is bidirectional. In that context, the hippocampal overactivity that develops with aging is a possible initiator and/or potentiator of pathological processes in $\mathrm{LOAD}$, as supported by recent evidence in humans [57]. The circular relationship between activity and amyloid, in particular, may initiate a vicious cycle of increasing hyperactivity driving greater pathology, supporting the perspective that aging itself plays a role in contributing to vulnerability for disease.

\section{MTL System and Cortical Networks in Aging, AD Models, and aMCI}

Biomarkers for preclinical AD have been detected in brain regions outside of the hippocampus proper, specifically in cortical regions of the MTL and circuits in the default mode network (DMN) that are strongly connected with the hippocampus. Beneficial effects of LEV have been reported not only on the targeted hyperactivity in the hippocampus, but extend to the broader MTL-cortical network in age-related impairment, as well as in mouse models of AD. This section highlights data that indicate certain parallels in the impairment-related alterations of the aging brain and vulnerability of specific sites to AD pathology within these extended MTL-cortical circuits, including beneficial effects of LEV treatment across a broad network.

\section{EC and MTL Circuitry}

The layer 2 neurons of the EC form the perforant path input to the DG/CA3 subfields of the hippocampal network, as schematically illustrated in Fig. 4(A). The layer 2 EC neurons are distinguished for their vulnerability in $\mathrm{AD}$, representing the earliest lesion affected by frank neurodegeneration. In autopsied brains well-characterized for amyloid and tau pathology, loss in the number of layer $2 \mathrm{EC}$ neurons can be detected in the prodromal $\mathrm{AD}$ phase of $\mathrm{MCI}$ (clinical dementia rating (CDR) 0.5 [1]), while no loss is detected in normal aging in the absence of AD pathology [114]. In other species, including rodents and nonhuman primates, numbers of neurons in the EC, and in layer 2 specifically, are preserved, even in aged animals with behaviorally assessed memory impairment [115-117]. However, a loss in the integrity of these neurons is evident across the spectrum of age-related memory impairment in laboratory animals and humans. An example of this altered condition involves the expression of reelin, which is a phenotypic marker of layer $2 \mathrm{EC}$ neurons across species $[118,119]$. In the adult brain, strong evidence demonstrates reelin contributes to normal synaptic function and plasticity [120, 121].

In aged rats with memory impairment both mRNA and protein expression of reelin in layer $2 \mathrm{EC}$ neurons is reduced, a condition that is rescued by LEV treatment [122]; Fig. 4B). In the brains of aged rhesus monkeys characterized for memory performance, reelin reduction in layer 2 EC is similarly observed in memory-impaired subjects with expression in aged unimpaired monkeys remaining on a par with young adults (P.R. Rapp, personal communication). Loss of EC reelin is also seen in mouse models of FAD and in patients with $\mathrm{AD}$, consistent with the well-known vulnerability of neurons in layer 2 of the EC [123, 124]. Given evidence for reelin in regulating excitability and synaptic plasticity, its loss in layer $2 \mathrm{EC}$ may alter not only the normal function of this key input pathway, but may contribute to the pathology of $\mathrm{A} \beta$ and tau in $\mathrm{AD}$. Indeed, in reelin heterozygous mutant mice crossed with familial $\mathrm{AD}$ transgenic mice, reelin reduction accelerates amyloid and tau pathology in hippocampal circuits [125] and has been co-localized with intracellular $A \beta$ in EC neurons in an AD rat model, as well as in early $\mathrm{AD}$ brains [126]. Recovery of reelin expression with LEV in aged subjects may indicate that this early-stage loss of neuronal integrity in the $\mathrm{EC}$ is a reversible condition.

As described in an earlier section of this review, a critical computation function on the EC input in the DG is to distinctively encode input patterns in a small number of granule cells. Mechanisms for sparse encoding in the DG are under local inhibitory control (illustrated in Fig. 4A). Selective targeting of DG inhibition can disrupt this function leading to greater neuronal activation by an input pattern and behavioral impairment on tasks with high mnemonic interference [64, 127]. In aged rats with memory impairment, stereological assessment of interneuron numbers found a reduction in a subpopulation of DG hilar inhibitory neurons that co-express somatostatin (SOM) relative to young rats and aged rats with unimpaired memory [128], while total numbers of neurons in the hilus were unchanged. This "HIPP" subpopulation, with cell bodies located in the hilus, innervates the molecular layer at the perforant path input from EC layer 2. Additional evidence for the relevance of SOM neuron loss in aging and risk for AD comes from studies of the human ApoE4 allele, a known risk factor for LOAD. ApoE4-positive individuals show elevated hippocampal activity during a memory task, suggesting this allele modifies excitatory/inhibitory balance [129]. 

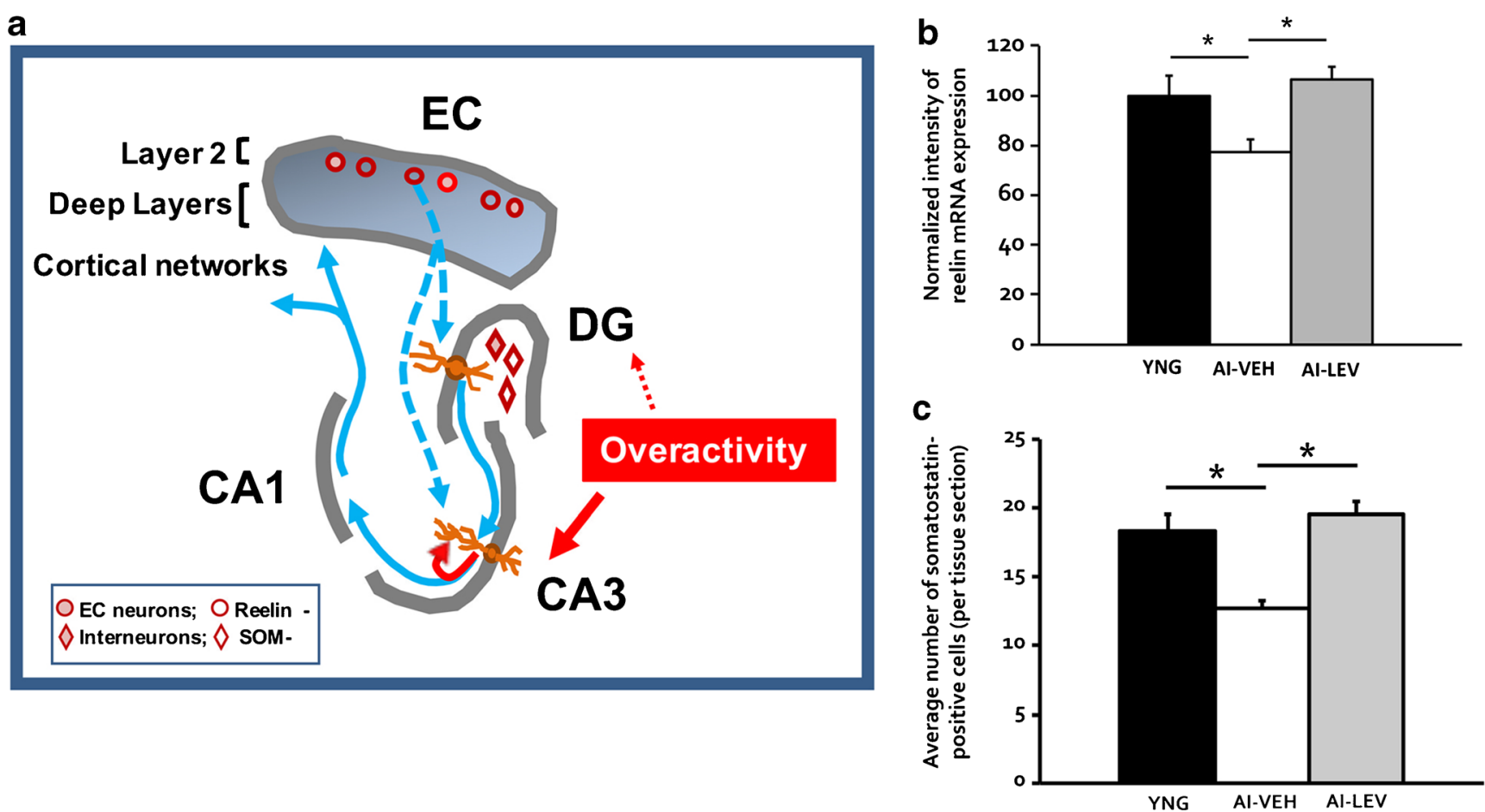

Fig. 4 Alterations coinciding with hippocampal hyperactivity and memory impairment. (A) Schematic illustrating the reduction of Reelin expression in entorhinal cortex (EC) layer 2 and somatostatin (SOM) expression in the dentate hilus interneurons in age-related memory impairment. (B) These data replicate the findings in Stranahan et al. [122] of reduced reelin mRNA in EC layer II neurons of aged impaired (AI) rats and its reversal in AI rats treated with levetiracetam (LEV) by osmotic minipump ( $10 \mathrm{mg} / \mathrm{kg} /$ day) for 28 days (figure panel provided by

Expression of the ApoE4 allele in mice exacerbated the agedependent loss of hilar SOM neurons relative to the riskneutral E3 allele [130]. Furthermore, E4 allele expression in this study contributed to greater memory impairment in older mice. The ApoE4 effect on SOM neurons potentially links aging and augmented risk for $\mathrm{AD}$ in carriers of the $\mathrm{E} 4$ allele. Notably, as shown in Fig. 4(C), similar to reelin expression in EC, SOM expression in HIPP interneurons in the hilus/DG was rescued by LEV treatment in memory-impaired aged rats [128].

In addition to these examples, other network-wide benefits of LEV treatment have been described in APP models of amyloid overexpression. As reviewed elsewhere [78, 103], $\mathrm{AD}$ pathology causes complex derangements of neuronal activity affecting extensive interconnected networks. Alongside aberrant excitatory activity, compensatory responses can also become engaged in an adaptive reorganization of the network around overactivity. A relatively widespread normalization in such network function has been observed by targeting hyperactivity in AD mouse models. For example, in hAPP-J20 mice treated with LEV, beneficial effects on synaptic plasticity in the DG were observed alongside a normalization of throughput, for example electrophysiological recording of input/ output functions, at CA3/CA1 synapses [106]. Such evidence for a network effect of LEV extends to patients with aMCI,
Koh, MT and Gallagher, M); $* p<0.05$. (C) Somatostatin expression in dentate hilus interneurons is reduced in AI rats and rescued by the same osmotic minipump treatment with LEV; ${ }^{*} p<0.05$. Part (C) adapted from Spiegel, A.M., Koh, M.T., Vogt, N.M., Rapp, P.R., \& Gallagher, M. Journal of Comparative Neurology vol 521, p. 3508-3523. Copyright (2013) reprinted with permission from John Wiley and Sons [128]. $\mathrm{DG}=$ dentate gyrus; $\mathrm{YNG}=$ young; $\mathrm{AI}-\mathrm{VEH}=\mathrm{AI}$ rats treated with saline vehicle; $\mathrm{AI}-\mathrm{LEV}=\mathrm{AI}$ rats treated with $\mathrm{LEV}$

who, alongside hippocampal overactivity, exhibit reduced fMRI activation in the EC (Fig. 3A) that is likewise reversed with LEV [20,21]. These data support a network perspective on both the condition of cognitive impairment and remediation of cognitive decline.

\section{Disruption of Function in Regions of the DMN}

Altered neural function associated with $\mathrm{AD}$ pathology and aging also occurs in cortical networks functionally interconnected with the MTL. In AD mouse models, $\mathrm{A} \beta$ associated hyperactivity is closely tied to corticocortical and corticohippocampal circuit disruptions that are suggested to impair memory function [77, 131]. Likewise, human imaging studies show altered functional connectivity across brain regions in elderly humans and those with aMCI [132-134], including early accumulation of high levels of amyloid pathology particularly evident in component structures of the DMN [132].

The DMN is a distributed cortical network defined by coordinated activity in a resting state that shows deactivation (reduced activity) in favor of task-specific network activation during cognitive engagement. Posterior components of DMN, including precuneus, posterior cingulate, retrosplenial cortex, and bilateral inferior parietal lobule, which exhibit strong functional 
connectivity with MTL, have been earlier described as a hippocampal-parietal memory network [135]. Altered functional connectivity across the DMN and reduced "deactivation" is evident with aging and cognitive impairment in clinical studies [136-138]. For example, a recent study of cognitively normal older individuals reported reduced DMN deactivation, localized to posterior cortical regions of the DMN, that occurred specifically in a subgroup of individuals that experienced cognitive decline based on their own longitudinal performance on neuropsychological assessments compared with individuals who had maintained cognitive abilities [138].

We recently demonstrated that alongside the CA3, evidence for heightened excitability is also present in posterior cortical regions interconnected with the hippocampus in aged memory-impaired rats. In response to pharmacological induction of neural activity, aged impaired rats exhibited elevated levels of cFos, relative to young and aged-unimpaired rats, in a subset of the structures that comprise the rat DMN, including posterior parietal cortex and retrosplenial cortices. In the impaired aged rats, elevated cFos was limited to specific cortical regions, with no excess elevation in the anterior cingulate cortex or thalamus (see Fig. 2B) [22]. Important in the current context, the elevated posterior cortical activity in aged impaired rats responded to a cognitively effective dose of LEV treatment, lowering induced cFos to the level of young rats. Notably, no effect of LEV in the aged impaired subjects was seen in any region that did not show initial heightened activation.

MTL deficits are recognized as contributing to the cognitive impairment observed in both aging and aMCI. Interest in the impact of $\mathrm{AD}$ on distributed neocortical networks has gained increasing attention, particularly since neuroimaging has shown deposition of cortical amyloid in preclinical AD. Early amyloid deposition is observed in DMN, including regions that are strongly interconnected with the hippocampus and MTL network $[132,139]$. Accumulated pathology has been associated with altered activity in MTL cortical regions interacting with the hippocampus in some studies [140].

In the case of cortical circuits, similar to the MTL, it is difficult to separate effects of aging from pathological processes in elderly humans owing to the lengthy preclinical phase of the disease superimposed upon an aging brain. Here, again, the use of a rodent model for age-related impairment, devoid of the natural occurrence, or genetically introduced, pathophysiology of $\mathrm{AD}$, has shown potentially important parallels between aging and AD pathology in a broad MTL/cortical network. Specifically, the intervention with LEV in the model of age-dependent memory impairment suggests that an altered condition may exist underlying the vulnerability of those circuits to early $\mathrm{AD}$ pathology. While the use of in vivo assessment has been integral to illuminating the affected circuitry in aging, further work is needed to address whether treatment effects are obtained by direct action of therapeutics on numerous distributed sites or whether widespread normalization of network function reflects both primary and secondary effects of targeting hyperactivity. In either case, a network perspective is needed to address the basis for impairment and understanding the effects of therapeutic intervention in aging that may extend to the progressive cognitive decline and accumulation of pathology in early LOAD.

\section{LEV Mechanism of Action Supports Successful Use as Therapy}

The atypical antiepileptic, LEV, was approved in 1999 as a second-generation drug and is most often used as an add-on therapy in the treatment of epilepsy [141]. Even at much higher chronic dosing as an adjunctive treatment in epilepsy, LEV has an excellent safety profile with few side effects in elderly patients $[66,142]$.

Mechanistically, LEV is well positioned to affect neuronal function in a beneficial manner in the condition of overactivity in aging and prodromal $\mathrm{AD}$ where efficacy has been reported with low-dose administration. LEV belongs to a class of compounds with high affinity for the presynaptic membrane protein SV2a, which is widely expressed throughout the brain, including high levels of expression in the hippocampus [143]. While the role of SV2a in biological function is not completely understood, strong evidence demonstrates a role in modulating calcium-dependent neurotransmitter release via multiple mechanisms with a greater effect during high activation [69, 144-146]. SV2A influences neurotransmitter release via expression and trafficking of the calcium sensor synaptotagmin and likely binds directly to synaptotagmin [145, 147]. SV2a also contributes to the mobilization of synaptic vesicles for release, and SV2a deletion reduces vesicle release during trains of action potentials but does not measurably affect steady-state activity $[144,146]$. LEV treatment has shown a beneficial effect in modulating neurotransmission in a number in vitro and in vivo models of elevated activity, and the binding of LEV to SV2a is likely a primary mechanism of action [24, $106,148]$.

Other beneficial effects of LEV have also been identified that improve neuronal dysfunction induced by AD pathology. In addition to mechanisms for quieting overactive neurons by limiting transmitter release, LEV has been demonstrated to inhibit both ryanodine and IP3 receptor-activated calcium release in hippocampal neurons [149, 150], which would be neuroprotective in the context of impaired calcium homeostasis in $\mathrm{AD}[151,152]$. Recent evidence of further interest has also shown beneficial effects of LEV on mitochondria, in which amyloid-associated mitochondrial dysfunction of fission and fusion imbalance was corrected by LEV [153]. In total, LEV treatment appears to have many beneficial effects on brain deficits associated with impaired cognitive function, 
induced by aging or $\mathrm{AD}$ pathology, with reduction of elevated neural activity most central to its effects.

\section{Targeting Hippocampal Hyperactivity to Stem Disease Progression}

With the number of Americans living with AD dementia expected to more than double by 2050 , it is predicted that development of a therapy that delays onset or slows progression to clinical dementia by even 1 to 2 years could reduce AD prevalence by $10 \%$ to $20 \%$ [154, 155]. In this review, we have presented evidence for the efficacy of targeting hippocampal hyperactivity using LEV as an initial therapeutic intervention. As a condition present in the earliest stages of $\mathrm{AD}$, reduction of hyperactivity has strong potential to affect the course of the disease and its rate of progression. While studies in animal models of aging and a phase II trial in aMCI support the case for targeting hyperactivity, clinical testing in a phase III trial is needed to ascertain the long-term effects of this treatment approach on progression. An appropriate phase of disease for such treatment could be MCI due to $\mathrm{AD}$, in which hippocampal hyperactivity is most pronounced. In addition to targeting hyperactivity as a monotherapy, given evidence for hyperactivity driving $\mathrm{AD}$ pathology, this treatment may also be useful in combination with other therapeutic approaches, such as amyloid-lowering therapies or treatments targeting tau.

\section{Conclusions}

Based on the evidence presented, we propose a model of LOAD whereby aging itself creates an environment in which abnormal, elevated neural activity promotes circuit dysfunction and contributes to the accumulation of pathology. In this view, the emergence of hyperactivity in the hippocampus and alterations in associated MTL circuits are key early events in progressive neural dysfunction. Indeed, recent investigations indicate that hippocampal hyperactivity may occur prior to and drive $A \beta$ accumulation. The mechanisms underlying the genesis of hyperactivity remain to be determined but likely involve alterations in excitatory/inhibitory balance, which may be driven by either expression of the ApoE4 allele or direct or indirect loss of inhibitory control. This hyperactivity appears to play a central role underlying further cognitive decline in prodromal LOAD. Interactions between hyperactivity and amyloid and tau pathophysiological pathways will also require further study to conclusively determine mechanistic, causal relationships. The data described here support a role for all 3 in the disruption of network function and cognitive decline. Elucidation of those mechanisms will provide further insight into how age-dependent elevation of neural activity contributes to cognitive decline and progression of disease in LOAD. For example, resolution of the mechanistic, spatial, and temporal relationships between cortical $\mathrm{A} \beta$ and hippocampal dysfunction will be critical in guiding the timing and selection of appropriate therapeutic strategies.

Key evidence in support of this proposal is the efficacy of LEV, a therapy that effectively targets abnormal neural activity, in ameliorating cognitive decline in both preclinical animal models and human aMCI subjects. Based on findings from transgenic $\mathrm{AD}$ models regarding a role for neural activity in driving amyloid and tau pathology, we expect that targeting hyperactivity in aMCI will not only improve cognition, but may also slow or prevent the accumulation of pathology and clinical decline. While LEV is currently being pursued as a potential therapeutic for $\mathrm{AD}$ prevention in a human clinical trial, other therapeutic approaches that restore balanced neural activity may also prove effective in preventing disease progression and accumulating AD pathology. Successful development of such therapies could have vast implications for achieving therapeutic treatment in LOAD to reduce the prevalence of patients with $\mathrm{AD}$ dementia.

Acknowledgments This work was supported by National Institute on Aging/National Institutes of Health program project grant P01AG009973-22, Postdoctoral Training grant T32 AG027668, and RO1 AG048349. We would like thank Ming Teng Koh for his contribution and assistance with the figures.

Open Access This article is distributed under the terms of the Creative Commons Attribution 4.0 International License (http:// creativecommons.org/licenses/by/4.0/), which permits unrestricted use, distribution, and reproduction in any medium, provided you give appropriate credit to the original author(s) and the source, provide a link to the Creative Commons license, and indicate if changes were made.

\section{References}

1. Albert MS, DeKosky ST, Dickson D, et al. The diagnosis of mild cognitive impairment due to Alzheimer's disease: recommendations from the National Institute on Aging-Alzheimer's Association workgroups on diagnostic guidelines for Alzheimer's disease. Alzheimers Dement 2011;7(3):270-279.

2. Petersen RC. Alzheimer's disease: progress in prediction. Lancet Neurol 2010;9(1):4-5.

3. Sperling RA, Aisen PS, Beckett LA, et al. Toward defining the preclinical stages of Alzheimer's disease: recommendations from the National Institute on Aging-Alzheimer's Association workgroups on diagnostic guidelines for Alzheimer's disease. Alzheimers Dement 2011;7(3):280-292.

4. Ringman JM, Goate A, Masters CL, et al. Genetic heterogeneity in Alzheimer disease and implications for treatment strategies. Curr Neurol Neurosci Rep 2014;14(11):499.

5. Holtzman DM, Morris JC, Goate AM. Alzheimer's disease: the challenge of the second century. Sci Transl Med 2011;3(77):77sr1.

6. Golde TE, Schneider LS, Koo EH. Anti-abeta therapeutics in Alzheimer's disease: the need for a paradigm shift. Neuron 2011;69(2):203-213. 
7. Sperling R, Mormino E, Johnson K. The evolution of preclinical Alzheimer's disease: implications for prevention trials. Neuron 2014;84(3):608-622.

8. Cavedo E, Lista S, Khachaturian Z, et al. The road ahead to cure Alzheimer's disease: development of biological markers and neuroimaging methods for prevention trials across all stages and target populations. J Prev Alzheimers Dis 2014;1(3):181-202.

9. Alzheimer's Association. 2010 Alzheimer's disease facts and figures. Alzheimers Dement 2010;6.

10. Dickerson BC, Salat DH, Greve DN, et al. Increased hippocampal activation in mild cognitive impairment compared to normal aging and AD. Neurology 2005;65(3):404-411.

11. Braak H, Braak E. Neuropathological stageing of Alzheimerrelated changes. Acta Neuropathol 1991;82(4):239-259.

12. Sperling R. Functional MRI studies of associative encoding in normal aging, mild cognitive impairment, and Alzheimer's disease. Ann N Y Acad Sci 2007;1097:146-155.

13. Dickerson BC, Sperling RA. Functional abnormalities of the medial temporal lobe memory system in mild cognitive impairment and Alzheimer's disease: insights from functional MRI studies. Neuropsychologia 2008;46(6):1624-1635.

14. Miller SL, Fenstermacher E, Bates J, Blacker D, Sperling RA, Dickerson BC. Hippocampal activation in adults with mild cognitive impairment predicts subsequent cognitive decline. J Neurol Neurosurg Psychiatry 2008;79(6):630-635.

15. Yassa MA, Stark SM, Bakker A, Albert MS, Gallagher M, Stark CE. High-resolution structural and functional MRI of hippocampal CA3 and dentate gyrus in patients with amnestic Mild Cognitive Impairment. Neuroimage 2010;51(3):1242-1252.

16. Sperling R. Potential of functional MRI as a biomarker in early Alzheimer's disease. Neurobiol Aging 2011;32(Suppl. 1):S37S43.

17. Putcha D, Brickhouse M, O'Keefe K, et al. Hippocampal hyperactivation associated with cortical thinning in Alzheimer's disease signature regions in non-demented elderly adults. J Neurosci 2011;31(48): 17680-17688.

18. Yassa MA, Lacy JW, Stark SM, Albert MS, Gallagher M, Stark CE. Pattern separation deficits associated with increased hippocampal CA3 and dentate gyrus activity in nondemented older adults. Hippocampus 2011;21(9):968-979.

19. O'Brien JL, O'Keefe KM, LaViolette PS, et al. Longitudinal fMRI in elderly reveals loss of hippocampal activation with clinical decline. Neurology 2010;74(24):1969-1976.

20. Bakker A, Krauss GL, Albert MS, et al. Reduction of hippocampal hyperactivity improves cognition in amnestic mild cognitive impairment. Neuron 2012;74(3):467-474.

21. Bakker A, Albert MS, Krauss G, Speck CL, Gallagher M. Response of the medial temporal lobe network in amnestic mild cognitive impairment to therapeutic intervention assessed by fMRI and memory task performance. Neuroimage Clin 2015;7: 688-698.

22. Haberman RP, Koh MT, Gallagher M. Heightened cortical excitability in aged rodents with memory impairment. Neurobiol Aging 2017;54:144-151.

23. Thome A, Gray DT, Erickson CA, Lipa P, Barnes CA. Memory impairment in aged primates is associated with region-specific network dysfunction. Mol Psychiatry 2016;21(9):1257-1262.

24. Koh MT, Haberman RP, Foti S, McCown TJ, Gallagher M. Treatment strategies targeting excess hippocampal activity benefit aged rats with cognitive impairment. Neuropsychopharmacology 2010;35(4):1016-1025.

25. Koh MT, Rosenzweig-Lipson S, Gallagher M. Selective GABA(A) alpha5 positive allosteric modulators improve cognitive function in aged rats with memory impairment. Neuropharmacology 2013;64:145-152.
26. Wilson IA, Ikonen S, McMahan RW, Gallagher M, Eichenbaum $\mathrm{H}$, Tanila H. Place cell rigidity correlates with impaired spatial learning in aged rats. Neurobiol Aging 2003;24(2):297-305.

27. Busche MA, Chen X, Henning HA, et al. Critical role of soluble amyloid-beta for early hippocampal hyperactivity in a mouse model of Alzheimer's disease. Proc Natl Acad Sci U S A 2012;109(22):8740-8745.

28. Busche MA, Konnerth A. Neuronal hyperactivity - a key defect in Alzheimer's disease? Bioessays 2015;37(6):624-632.

29. Bero AW, Yan P, Roh JH, et al. Neuronal activity regulates the regional vulnerability to amyloid-beta deposition. Nat Neurosci 2011;14(6):750-756.

30. Yuan P, Grutzendler J. Attenuation of beta-amyloid deposition and neurotoxicity by chemogenetic modulation of neural activity. $\mathrm{J}$ Neurosci 2016;36(2):632-641.

31. Wu JW, Hussaini SA, Bastille IM, et al. Neuronal activity enhances tau propagation and tau pathology in vivo. Nat Neurosci 2016;19(8):1085-1092.

32. Rapp PR. Aging and memory in animals. In: Squire LR, editor. Encyclopedia of neuroscience. Oxford: Academic Press; 2009. pp. 167-174.

33. Gallagher M, Burwell R, Burchinal M. Severity of spatial learning impairment in aging: development of a learning index for performance in the Morris water maze. Behav Neurosci 1993;107(4): 618-626.

34. Gallagher M, Stocker AM, Koh MT. Mindspan: lessons from rat models of neurocognitive aging. ILAR J 2011;52(1):32-40.

35. Wilson IA, Ikonen S, Gallagher M, Eichenbaum H, Tanila H. Ageassociated alterations of hippocampal place cells are subregion specific. J Neurosci 2005;25(29):6877-6886.

36. Knierim JJ, Neunuebel JP. Tracking the flow of hippocampal computation: pattern separation, pattern completion, and attractor dynamics. Neurobiol Learn Mem 2016;129:38-49.

37. Leutgeb JK, Leutgeb S, Moser MB, Moser EI. Pattern separation in the dentate gyrus and CA3 of the hippocampus. Science 2007;315(5814):961-966.

38. Neunuebel JP, Knierim JJ. CA3 retrieves coherent representations from degraded input: direct evidence for CA3 pattern completion and dentate gyrus pattern separation. Neuron 2014;81(2):416-427.

39. Treves A, Rolls ET. Computational constraints suggest the need for two distinct input systems to the hippocampal CA3 network. Hippocampus 1992;2(2):189-199.

40. Rolls ET, Kesner RP. A computational theory of hippocampal function, and empirical tests of the theory. Prog Neurobiol 2006;79(1):1-48.

41. Bakker A, Kirwan CB, Miller M, Stark CE. Pattern separation in the human hippocampal CA3 and dentate gyrus. Science 2008;319(5870):1640-1642.

42. Yassa MA, Stark CE. Pattern separation in the hippocampus. Trends Neurosci 2011;34(10):515-525.

43. Wilson IA, Gallagher M, Eichenbaum H, Tanila H. Neurocognitive aging: prior memories hinder new hippocampal encoding. Trends Neurosci 2006;29(12):662-670.

44. Leal SL, Yassa MA. Neurocognitive aging and the hippocampus across species. Trends Neurosci 2015;38(12):800-812.

45. Kirwan CB, Stark CE. Overcoming interference: an fMRI investigation of pattern separation in the medial temporal lobe. Learn Mem 2007;14(9):625-633.

46. Stark SM, Yassa MA, Lacy JW, Stark CE. A task to assess behavioral pattern separation (BPS) in humans: Data from healthy aging and mild cognitive impairment. Neuropsychologia 2013;51(12): 2442-2449.

47. Toner CK, Pirogovsky E, Kirwan CB, Gilbert PE. Visual object pattern separation deficits in nondemented older adults. Learn Mem 2009;16(5):338-342. 
48. Holden HM, Hoebel C, Loftis K, Gilbert PE. Spatial pattern separation in cognitively normal young and older adults. Hippocampus 2012;22(9):1826-1832.

49. Holden HM, Toner C, Pirogovsky E, Kirwan CB, Gilbert PE. Visual object pattern separation varies in older adults. Learn Mem 2013;20(7):358-362.

50. Robitsek RJ, Fortin NJ, Koh MT, Gallagher M, Eichenbaum H. Cognitive aging: a common decline of episodic recollection and spatial memory in rats. J Neurosci 2008;28(36):8945-8954.

51. Celone KA, Calhoun VD, Dickerson BC, et al. Alterations in memory networks in mild cognitive impairment and Alzheimer's disease: an independent component analysis. J Neurosci 2006;26(40):10222-10231.

52. Dickerson BC, Salat DH, Bates JF, et al. Medial temporal lobe function and structure in mild cognitive impairment. Ann Neurol 2004;56(1):27-35.

53. Hamalainen A, Pihlajamaki M, Tanila $\mathrm{H}$, et al. Increased fMRI responses during encoding in mild cognitive impairment. Neurobiol Aging 2007;28(12):1889-1903.

54. Tran TT, Speck CL, Pisupati A, Gallagher M, Bakker A. Increased hippocampal activation in ApoE-4 carriers and non-carriers with amnestic mild cognitive impairment. Neuroimage Clin 2017;13: 237-245.

55. Ewers M, Sperling RA, Klunk WE, Weiner MW, Hampel H. Neuroimaging markers for the prediction and early diagnosis of Alzheimer's disease dementia. Trends Neurosci 2011;34(8):430442 .

56. Huijbers W, Mormino EC, Schultz AP, et al. Amyloid-beta deposition in mild cognitive impairment is associated with increased hippocampal activity, atrophy and clinical progression. Brain 2015;138(Pt 4):1023-1035.

57. Leal SL, Landau S, Bell R, Jagust W. Hippocampal activation is associated with longitudinal amyloid accumulation and cognitive decline. eLife 2017;6:e22978

58. Robitsek J, Ratner MH, Stewart T, Eichenbaum H, Farb DH. Combined administration of levetiracetam and valproic acid attenuates age-related hyperactivity of CA3 place cells, reduces place field area, and increases spatial information content in aged rat hippocampus. Hippocampus 2015;25(12):1541-1555.

59. Sur C, Fresu L, Howell O, McKernan RM, Atack JR. Autoradiographic localization of alpha5 subunit-containing GABAA receptors in rat brain. Brain Res 1999;822(1-2):265270.

60. Chambers MS, Atack JR, Broughton HB, et al. Identification of a novel, selective GABA(A) alpha5 receptor inverse agonist which enhances cognition. J Med Chem 2003;46(11):2227-2240.

61. Atack JR, Bayley PJ, Seabrook GR, Wafford KA, McKernan RM, Dawson GR. L-655,708 enhances cognition in rats but is not proconvulsant at a dose selective for alpha5-containing GABAA receptors. Neuropharmacology 2006;51(6):1023-1029.

62. Collinson N, Atack JR, Laughton P, Dawson GR, Stephens DN. An inverse agonist selective for alpha5 subunit-containing GABAA receptors improves encoding and recall but not consolidation in the Morris water maze. Psychopharmacology (Berl) 2006;188(4):619-628.

63. Dawson GR, Maubach KA, Collinson N, et al. An inverse agonist selective for alpha5 subunit-containing GABAA receptors enhances cognition. J Pharmacol Exp Ther 2006;316(3):1335-1345.

64. Engin E, Zarnowska ED, Benke D, et al. Tonic inhibitory control of dentate gyrus granule cells by alpha5-containing GABAA receptors reduces memory interference. J Neurosci 2015;35(40): 13698-13712.

65. Cumbo E, Ligori LD. Levetiracetam, lamotrigine, and phenobarbital in patients with epileptic seizures and Alzheimer's disease. Epilepsy Behav 2010;17(4):461-466.
66. Cramer JA, Leppik IE, Rue KD, Edrich P, Kramer G. Tolerability of levetiracetam in elderly patients with CNS disorders. Epilepsy Res 2003;56(2-3):135-145.

67. Contin M, Mohamed S, Albani F, Riva R, Baruzzi A. Levetiracetam clinical pharmacokinetics in elderly and very elderly patients with epilepsy. Epilepsy Res 2012;98(2-3):130-134

68. Meehan AL, Yang X, McAdams BD, Yuan L, Rothman SM. A new mechanism for antiepileptic drug action: vesicular entry may mediate the effects of levetiracetam. J Neurophysiol 2011;106(3): 1227-1239.

69. Lynch BA, Lambeng N, Nocka K, et al. The synaptic vesicle protein SV2A is the binding site for the antiepileptic drug levetiracetam. Proc Natl Acad Sci U S A 2004;101(26):9861-9866.

70. Yang XF, Rothman SM. Levetiracetam has a time- and stimulation-dependent effect on synaptic transmission. Seizure 2009;18(9):615-619.

71. Jagust W. Vulnerable neural systems and the borderland of brain aging and neurodegeneration. Neuron 2013;77(2):219-234.

72. Gotz J, Ittner LM. Animal models of Alzheimer's disease and frontotemporal dementia. Nat Rev Neurosci 2008;9(7):532-544.

73. Elder GA, Gama Sosa MA, De Gasperi R. Transgenic mouse models of Alzheimer's disease. Mt Sinai J Med 2010;77(1):69-81.

74. Hsia AY, Masliah E, McConlogue L, et al. Plaque-independent disruption of neural circuits in Alzheimer's disease mouse models. Proc Natl Acad Sci U S A 1999;96(6):3228-3233.

75. Chapman PF, White GL, Jones MW, et al. Impaired synaptic plasticity and learning in aged amyloid precursor protein transgenic mice. Nat Neurosci 1999;2(3):271-276.

76. Palop JJ, Jones B, Kekonius L, et al. Neuronal depletion of calcium-dependent proteins in the dentate gyrus is tightly linked to Alzheimer's disease-related cognitive deficits. Proc Natl Acad Sci U S A 2003;100(16):9572-9577.

77. Palop JJ, Chin J, Roberson ED, et al. Aberrant excitatory neuronal activity and compensatory remodeling of inhibitory hippocampal circuits in mouse models of Alzheimer's disease. Neuron 2007;55(5):697-711.

78. Palop JJ, Mucke L. Amyloid-beta-induced neuronal dysfunction in Alzheimer's disease: from synapses toward neural networks. Nat Neurosci 2010;13(7):812-818.

79. Kobayashi DT, Chen KS. Behavioral phenotypes of amyloidbased genetically modified mouse models of Alzheimer's disease. Genes Brain Behav 2005;4(3):173-196.

80. Wilke SA, Raam T, Antonios JK, et al. Specific disruption of hippocampal mossy fiber synapses in a mouse model of familial Alzheimer's disease. PLOS ONE 2014;9(1):e84349.

81. Higuchi M, Zhang B, Forman MS, Yoshiyama Y, Trojanowski JQ, Lee VM. Axonal degeneration induced by targeted expression of mutant human tau in oligodendrocytes of transgenic mice that model glial tauopathies. J Neurosci 2005;25(41):9434-9443.

82. Forman MS, Lal D, Zhang B, et al. Transgenic mouse model of tau pathology in astrocytes leading to nervous system degeneration. J Neurosci 2005;25(14):3539-3550.

83. Allen B, Ingram E, Takao M, et al. Abundant tau filaments and nonapoptotic neurodegeneration in transgenic mice expressing human P301S tau protein. J Neurosci 2002;22(21):9340-9351.

84. Yoshiyama Y, Higuchi M, Zhang B, et al. Synapse loss and microglial activation precede tangles in a P301S tauopathy mouse model. Neuron 2007;53(3):337-351.

85. Bellucci A, Westwood AJ, Ingram E, Casamenti F, Goedert M, Spillantini MG. Induction of inflammatory mediators and microglial activation in mice transgenic for mutant human P301S tau protein. Am J Pathol 2004;165(5):1643-1652.

86. Musiek ES, Holtzman DM. Three dimensions of the amyloid hypothesis: time, space and 'wingmen'. Nat Neurosci 2015;18(6): 800-806. 
87. Borchelt DR, Thinakaran G, Eckman CB, et al. Familial Alzheimer's disease-linked presenilin 1 variants elevate Abeta142/1-40 ratio in vitro and in vivo. Neuron 1996;17(5):1005-1013.

88. Duff K, Eckman C, Zehr C, et al. Increased amyloid-beta42(43) in brains of mice expressing mutant presenilin 1. Nature 1996;383(6602):710-713.

89. Lesne S, Koh MT, Kotilinek L, et al. A specific amyloid-beta protein assembly in the brain impairs memory. Nature 2006;440(7082):352-357.

90. Suberbielle E, Sanchez PE, Kravitz AV, et al. Physiologic brain activity causes DNA double-strand breaks in neurons, with exacerbation by amyloid-beta. Nat Neurosci 2013;16(5):613-621.

91. Minkeviciene R, Rheims S, Dobszay MB, et al. Amyloid betainduced neuronal hyperexcitability triggers progressive epilepsy. $\mathrm{J}$ Neurosci 2009;29(11):3453-3462.

92. Vogt DL, Thomas D, Galvan V, Bredesen DE, Lamb BT, Pimplikar SW. Abnormal neuronal networks and seizure susceptibility in mice overexpressing the APP intracellular domain. Neurobiol Aging 2011;32(9):1725-1729.

93. Roberson ED, Halabisky B, Yoo JW, et al. Amyloid-beta/Fyninduced synaptic, network, and cognitive impairments depend on tau levels in multiple mouse models of Alzheimer's disease. J Neurosci 2011;31(2):700-711.

94. Velez-Pardo C, Arellano JI, Cardona-Gomez P, Jimenez Del Rio M, Lopera F, De Felipe J. CA1 hippocampal neuronal loss in familial Alzheimer's disease presenilin-1 E280A mutation is related to epilepsy. Epilepsia 2004;45(7):751-756.

95. Noebels J. A perfect storm: Converging paths of epilepsy and Alzheimer's dementia intersect in the hippocampal formation. Epilepsia 2011;52(Suppl. 1):39-46.

96. Hazra A, Gu F, Aulakh A, Berridge C, Eriksen JL, Ziburkus J. Inhibitory neuron and hippocampal circuit dysfunction in an aged mouse model of Alzheimer's disease. PLOS ONE 2013;8(5): e64318.

97. Busche MA, Eichhoff G, Adelsberger H, et al. Clusters of hyperactive neurons near amyloid plaques in a mouse model of Alzheimer's disease. Science 2008;321(5896):1686-1689.

98. Grienberger C, Rochefort NL, Adelsberger H, et al. Staged decline of neuronal function in vivo in an animal model of Alzheimer's disease. Nat Commun 2012;3:774.

99. Rudinskiy N, Hawkes JM, Betensky RA, et al. Orchestrated experience-driven Arc responses are disrupted in a mouse model of Alzheimer's disease. Nat Neurosci 2012;15(10):1422-1429.

100. Siskova Z, Justus D, Kaneko H, et al. Dendritic structural degeneration is functionally linked to cellular hyperexcitability in a mouse model of Alzheimer's disease. Neuron 2014;84(5):10231033.

101. Vossel KA, Beagle AJ, Rabinovici GD, et al. Seizures and epileptiform activity in the early stages of Alzheimer disease. JAMA Neurol 2013;70(9):1158-1166.

102. Vossel KA, Ranasinghe KG, Beagle AJ, et al. Incidence and impact of subclinical epileptiform activity in Alzheimer's disease. Ann Neurol 2016;80(6):858-870.

103. Palop JJ, Mucke L. Network abnormalities and interneuron dysfunction in Alzheimer disease. Nat Rev Neurosci 2016;17(12): 777-792.

104. Cirrito JR, Kang JE, Lee J, et al. Endocytosis is required for synaptic activity-dependent release of amyloid-beta in vivo. Neuron 2008;58(1):42-51.

105. Roth BL. DREADDs for neuroscientists. Neuron 2016;89(4):683694.

106. Sanchez PE, Zhu L, Verret L, et al. Levetiracetam suppresses neuronal network dysfunction and reverses synaptic and cognitive deficits in an Alzheimer's disease model. Proc Natl Acad Sci U S A 2012;109(42):E2895-E2903.
107. Hall AM, Throesch BT, Buckingham SC, et al. Tau-dependent Kv4.2 depletion and dendritic hyperexcitability in a mouse model of Alzheimer's disease. J Neurosci 2015;35(15):6221-6230.

108. Tabuchi M, Lone SR, Liu S, et al. Sleep interacts with abeta to modulate intrinsic neuronal excitability. Curr Biol 2015;25(6): 702-712.

109. Shi JQ, Wang BR, Tian YY, et al. Antiepileptics topiramate and levetiracetam alleviate behavioral deficits and reduce neuropathology in APPswe/PS1dE9 transgenic mice. CNS Neurosci Ther 2013;19(11):871-881.

110. Nygaard HB, Kaufman AC, Sekine-Konno T, et al. Brivaracetam, but not ethosuximide, reverses memory impairments in an Alzheimer's disease mouse model. Alzheimers Res Ther 2015;7(1):25.

111. Roberson ED, Scearce-Levie K, Palop JJ, et al. Reducing endogenous tau ameliorates amyloid beta-induced deficits in an Alzheimer's disease mouse model. Science 2007;316(5825):750754.

112. Wegmann S, Maury EA, Kirk MJ, et al. Removing endogenous tau does not prevent tau propagation yet reduces its neurotoxicity. EMBO J 2015;34(24):3028-3041.

113. Maeda S, Djukic B, Taneja P, et al. Expression of A152T human tau causes age-dependent neuronal dysfunction and loss in transgenic mice. EMBO Rep 2016;17(4):530-551.

114. Gomez-Isla T, Hollister R, West $\mathrm{H}$, et al. Neuronal loss correlates with but exceeds neurofibrillary tangles in Alzheimer's disease. Ann Neurol 1997;41(1):17-24.

115. Rapp PR, Deroche PS, Mao Y, Burwell RD. Neuron number in the parahippocampal region is preserved in aged rats with spatial learning deficits. Cereb Cortex 2002;12(11):1171-1179.

116. Merrill DA, Roberts JA, Tuszynski MH. Conservation of neuron number and size in entorhinal cortex layers II, III, and V/VI of aged primates. J Comp Neurol 2000;422(3):396-401.

117. Gazzaley AH, Thakker MM, Hof PR, Morrison JH. Preserved number of entorhinal cortex layer II neurons in aged macaque monkeys. Neurobiol Aging 1997;18(5):549-553.

118. Perez-Garcia CG, Gonzalez-Delgado FJ, Suarez-Sola ML, et al. Reelin-immunoreactive neurons in the adult vertebrate pallium. J Chem Neuroanat 2001;21(1):41-51.

119. Ramos-Moreno T, Galazo MJ, Porrero C, Martinez-Cerdeno V, Clasca F. Extracellular matrix molecules and synaptic plasticity: immunomapping of intracellular and secreted Reelin in the adult rat brain. Eur J Neurosci 2006;23(2):401-422.

120. Beffert U, Weeber EJ, Durudas A, et al. Modulation of synaptic plasticity and memory by Reelin involves differential splicing of the lipoprotein receptor Apoer2. Neuron 2005;47(4):567-579.

121. Herz J, Chen Y. Reelin, lipoprotein receptors and synaptic plasticity. Nat Rev Neurosci 2006;7(11):850-859.

122. Stranahan AM, Haberman RP, Gallagher M. Cognitive decline is associated with reduced reelin expression in the entorhinal cortex of aged rats. Cereb Cortex 2011;21(2):392-400.

123. Chin J, Massaro CM, Palop JJ, et al. Reelin depletion in the entorhinal cortex of human amyloid precursor protein transgenic mice and humans with Alzheimer's disease. J Neurosci 2007;27(11): 2727-2733.

124. Herring A, Donath A, Steiner KM, et al. Reelin depletion is an early phenomenon of Alzheimer's pathology. J Alzheimers Dis 2012;30(4):963-979.

125. Kocherhans S, Madhusudan A, Doehner J, et al. Reduced Reelin expression accelerates amyloid-beta plaque formation and tau pathology in transgenic Alzheimer's disease mice. J Neurosci 2010;30(27):9228-9240

126. Kobro-Flatmoen A, Nagelhus A, Witter MP. Reelinimmunoreactive neurons in entorhinal cortex layer II selectively express intracellular amyloid in early Alzheimer's disease. Neurobiol Dis 2016;93:172-183. 
127. Andrews-Zwilling Y, Gillespie AK, Kravitz AV, et al. Hilar GABAergic interneuron activity controls spatial learning and memory retrieval. PLOS ONE 2012;7(7):e40555.

128. Spiegel AM, Koh MT, Vogt NM, Rapp PR, Gallagher M. Hilar interneuron vulnerability distinguishes aged rats with memory impairment. J Comp Neurol 2013;521(15):3508-3523.

129. Bookheimer SY, Strojwas MH, Cohen MS, et al. Patterns of brain activation in people at risk for Alzheimer's disease. N Engl J Med 2000;343(7):450-456.

130. Andrews-Zwilling Y, Bien-Ly N, Xu Q, et al. Apolipoprotein E4 causes age- and Tau-dependent impairment of GABAergic interneurons, leading to learning and memory deficits in mice. $\mathrm{J}$ Neurosci 2010;30(41):13707-13717.

131. Busche MA, Kekus M, Adelsberger H, et al. Rescue of long-range circuit dysfunction in Alzheimer's disease models. Nat Neurosci 2015;18(11):1623-1630.

132. Sperling RA, Laviolette PS, O'Keefe K, et al. Amyloid deposition is associated with impaired default network function in older persons without dementia. Neuron 2009;63(2):178-188.

133. Jones DT, Machulda MM, Vemuri P, et al. Age-related changes in the default mode network are more advanced in Alzheimer disease. Neurology 2011;77(16):1524-1531.

134. Andrews-Hanna JR, Snyder AZ, Vincent JL, et al. Disruption of large-scale brain systems in advanced aging. Neuron 2007;56(5): 924-935.

135. Vincent JL, Snyder AZ, Fox MD, et al. Coherent spontaneous activity identifies a hippocampal-parietal memory network. J Neurophysiol 2006;96(6):3517-3531.

136. Miller SL, Celone K, DePeau K, et al. Age-related memory impairment associated with loss of parietal deactivation but preserved hippocampal activation. Proc Natl Acad Sci U S A.2008;105(6):2181-2186.

137. Pihlajamaki M, Sperling RA. Functional MRI assessment of taskinduced deactivation of the default mode network in Alzheimer's disease and at-risk older individuals. Behav Neurol 2009;21(1): 77-91.

138. Hansen NL, Lauritzen M, Mortensen EL, et al. Subclinical cognitive decline in middle-age is associated with reduced task-induced deactivation of the brain's default mode network. Hum Brain Mapp 2014;35(9):4488-4498.

139. Jones DT, Knopman DS, Gunter JL, et al. Cascading network failure across the Alzheimer's disease spectrum. Brain 2016;139(Pt 2):547-562.

140. Huijbers W, Mormino EC, Wigman SE, et al. Amyloid deposition is linked to aberrant entorhinal activity among cognitively normal older adults. J Neurosci 2014;34(15):5200-5210.

141. Mendoza-Torreblanca JG, Vanoye-Carlo A, Phillips-Farfan BV, Carmona-Aparicio L, Gomez-Lira G. Synaptic vesicle protein 2A: basic facts and role in synaptic function. Eur J Neurosci 2013;38(11):3529-3539.
142. French J, Edrich P, Cramer JA. A systematic review of the safety profile of levetiracetam: a new antiepileptic drug. Epilepsy Res 2001;47(1-2):77-90.

143. Crevecoeur J, Foerch P, Doupagne M, et al. Expression of SV2 isoforms during rodent brain development. BMC Neurosci 2013;14:87.

144. Crowder KM, Gunther JM, Jones TA, et al. Abnormal neurotransmission in mice lacking synaptic vesicle protein 2A (SV2A). Proc Natl Acad Sci U S A 1999;96(26):15268-15273.

145. Nowack A, Yao J, Custer KL, Bajjalieh SM. SV2 regulates neurotransmitter release via multiple mechanisms. Am J Physiol Cell Physiol 2010;299(5):C960-C967.

146. Custer KL, Austin NS, Sullivan JM, Bajjalieh SM. Synaptic vesicle protein 2 enhances release probability at quiescent synapses. J Neurosci 2006;26(4):1303-1313.

147. Yao J, Nowack A, Kensel-Hammes P, Gardner RG, Bajjalieh SM. Cotrafficking of SV2 and synaptotagmin at the synapse. J Neurosci 2010;30(16):5569-5578.

148. Surges R, Volynski KE, Walker MC. Is levetiracetam different from other antiepileptic drugs? Levetiracetam and its cellular mechanism of action in epilepsy revisited. Ther Adv Neurol Disord 2008;1(1):13-24.

149. Nagarkatti N, Deshpande LS, DeLorenzo RJ. Levetiracetam inhibits both ryanodine and IP3 receptor activated calcium induced calcium release in hippocampal neurons in culture. Neurosci Lett 2008;436(3):289-293.

150. Cataldi M, Lariccia V, Secondo A, di Renzo G, Annunziato L. The antiepileptic drug levetiracetam decreases the inositol 1,4,5-trisphosphate-dependent [Ca2+]I increase induced by ATP and bradykinin in PC12 cells. J Pharmacol Exp Ther 2005;313(2):720730 .

151. Kuchibhotla KV, Goldman ST, Lattarulo CR, Wu HY, Hyman BT, Bacskai BJ. Abeta plaques lead to aberrant regulation of calcium homeostasis in vivo resulting in structural and functional disruption of neuronal networks. Neuron 2008;59:214-225.

152. Hermes M, Eichhoff G, Garaschuk O. Intracellular calcium signalling in Alzheimer's disease. J Cell Mol Med 2010;14(1-2):30-41.

153. Stockburger C, Miano D, Baeumlisberger M, et al. A mitochondrial role of SV2a protein in aging and Alzheimer's disease: studies with levetiracetam. J Alzheimers Dis 2016;50(1):201-215.

154. Brookmeyer R, Johnson E, Ziegler-Graham K, Arrighi HM. Forecasting the global burden of Alzheimer's disease. Alzheimers Dement 2007;3(3):186-191.

155. Alzheimer's Association. Changing the trajectory of Alzheimer's disease: How a treatment by 2025 saves lives and dollars. Available at: http://www.alz.org/documents_custom/trajectory. pdf. Accessed December 2016. 